\title{
Head and Neck Sebaceous Carcinoma
}

National Cancer Institute

\section{Source}

National Cancer Institute. Head and Neck Sebaceous Carcinoma. NCI Thesaurus. Code C160978.

A sebaceous carcinoma that arises from the skin in the head and neck. 